\title{
A PANOPLY OF CEPHEID LIGHT CURVE TEMPLATES
}

\author{
Peter Yoachim $^{1}$, Les P. McCommas ${ }^{2}$, Julianne J. Dalcanton ${ }^{2}$, and Benjamin F. Williams ${ }^{2}$ \\ ${ }^{1}$ Department of Astronomy and McDonald Observatory, University of Texas, Austin, TX 78712, USA; yoachim@ astro.as.utexas.edu \\ ${ }^{2}$ Department of Astronomy, University of Washington, Box 351580, Seattle, WA 98195, USA \\ Received 2008 November 7; accepted 2009 March 16; published 2009 April 23
}

\begin{abstract}
We have generated accurate $V$ and $I$ template light curves using a combination of Fourier decomposition and principal component analysis for a large sample of Cepheid light curves. Unlike previous studies, we include short-period Cepheids and stars pulsating in the first overtone mode in our analysis. Extensive Monte Carlo simulations show that our templates can be used to precisely measure Cepheid magnitudes and periods, even in cases where there are few observational epochs. These templates are ideal for characterizing serendipitously discovered Cepheids and can be used in conjunction with surveys such as Pan-Starrs and LSST where the observational sampling may not be optimized for Cepheids.
\end{abstract}

Key words: Cepheids - distance scale - stars: general

\section{INTRODUCTION}

The Cepheid period-luminosity (PL) relation is a fundamental rung in the astronomical distance ladder. With the Hubble Space Telescope (HST) routinely resolving stellar populations in nearby galaxies, Cepheid distances can potentially be calculated for a large number of new systems. In this paper, we show that high-quality template light curves can accurately fit pulsation periods and mean luminosities to sparsely sampled Cepheid observations. It is our hope that these templates can be used to characterize Cepheids in archival as well as new observations, even when the time sampling of observations has not been optimized for Cepheid studies.

Principal component analysis (PCA) has found application in a wide range of astronomical studies (e.g., Faber 1973; Conselice 2006). The motivation behind PCA is that it can greatly reduce the number of variables required to describe a data set. By performing an eigenvalue decomposition, PCA can be used to generate a small number of eigenvectors that describe the majority of the variance in a data set. In the case of Cepheid variables, one could use PCA analysis to construct light curves with the smallest possible number of free parameters. Ideally, all Cepheids with identical periods would have a single unique light-curve shape. In this case, only a period, an average magnitude, and a phase would be needed as free parameters to fit a light curve.

The classical technique for determining a stellar pulsation period is to calculate a "string length" (Lafler \& Kinman 1965; Burke et al. 1970). A light curve is folded along a trial period and a string length is computed by summing distances between points consecutive in phase. The trial period with the shortest computed string length is taken as the true period. This method is accurate for well-sampled light curves with small photometric errors, but has been supplanted by recent techniques.

More robust periods can be obtained by fitting template light curves. Fourier analysis of Cepheids was introduced in Schaltenbrand \& Tammann (1971), and Stetson (1996) generated templates based on Fourier decomposition of Milky Way (MW) and Large Magellanic Cloud (LMC) stars. Tanvir et al. (2005) used PCA to construct a large set of well-sampled Cepheid observations. Deb \& Singh (2009) use the Fourier plus PCA technique on many types of variable stars and show how powerful the technique can be for identifying different variable types. In this paper, we extend the techniques of Tanvir et al. (2005) to include short-period Cepheids as well as first overtone Cepheids.

Short-period Cepheids (with periods less than $\sim 10$ days) have been excluded from many studies for a variety of reasons. First, LMC Cepheids may show a discontinuity in the PL relation at 10 days, casting uncertainty on their utility as reliable standard candles (Ngeow \& Kanbur 2008). Second, there is the possibility of confusion between fundamental and first-overtone mode Cepheids that have similar periods but different PL relations. Third, if fainter stars like short-period Cepheids are used to estimate distances, incompleteness bias can skew the derived distances to smaller values (Sandage \& Carlson 1988; Freedman et al. 2001). Finally, the shorter period Cepheids show more variation in light-curve shape, making template construction more daunting. Keeping these potential problems in mind, we boldly go forward and derive short-period templates regardless.

The outline of the paper is as follows. In Section 2, we describe generating template light curves using PCA from a large literature sample of Cepheid stars. In Section 3, we perform Monte Carlo simulations to determine how precisely we can recover Cepheid parameters using our templates. In Section 4, we discuss the process of converting fit parameters into a distance measure.

\section{PRINCIPAL COMPONENT ANALYSIS}

\subsection{Training Set Selection}

Following Tanvir et al. (2005), we perform PCA on $V$ and $I$-band light curves simultaneously. We therefore gathered light curves for stars that have both $V$ and $I$ measurements. The majority of our template stars come from the Optical Gravitational Lensing Experiment (OGLE) databases for the LMC (Udalski et al. 1999a) and Small Magellanic Cloud (SMC; Udalski et al. 1999b). We gathered additional LMC observations from Sebo et al. (2002) and additional LMC and SMC light curves from Moffett et al. (1998). Our Galactic Cepheid sample was compiled from light curves in the VizieR database (Ochsenbein et al. 2000), including data presented in Berdnikov (1997) and Berdnikov \& Turner (2001). We also included Galactic Cepheids from the McMaster Cepheid 


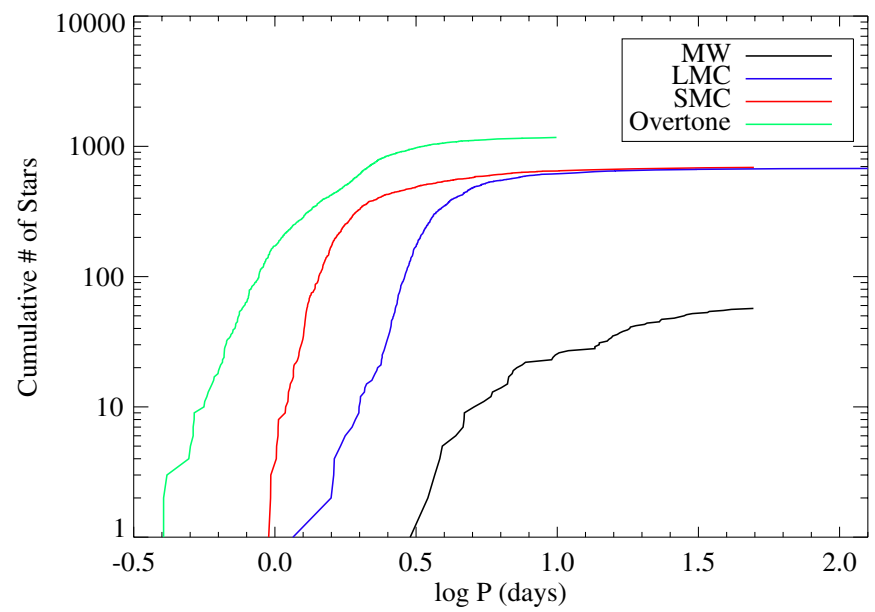

Figure 1. Period distribution of the stars that are used to generate our templates. The MW, LMC, and SMC fundamental mode stars along with the combined LMC and SMC first overtone pulsators are plotted.

Photometry and Radial Velocity Data Archive that includes light curves from many sources (Gieren 1981; Moffett \& Barnes 1984; Coulson \& Caldwell 1985; Berdnikov \& Turner 1995; Henden 1996; Barnes et al. 1997). We would have liked to include light curves from the MACHO survey. However, the MACHO survey uses nontraditional "blue" and "red" filters that are nontrivial to convert to $V$ and $I$.

To be included in our analysis we required each light curve to have at least 15 epochs of observations in both $V$ and $I$, with the exception of well-spaced light curves from Berdnikov \& Turner (2001), where we only demand six epochs in both $V$ and $I$. The initial sample contained 3173 light curves, with 248 Milky Way LCs, 1378 LMC LCs, and 1514 SMC LCs. Unlike the majority of previous Cepheid studies, we did not exclude stars with periods shorter than 10 days from the analysis. We fit Fourier components to every light curve and rejected 305 outliers (i.e., those with unusual light-curve shapes or poor fits). The final sample included $150 \mathrm{MW}$ Cepheids, $677 \mathrm{LMC}$ Cepheids, 689 SMC Cepheids, and 1171 overtone pulsators from the LMC (549 stars), SMC (575 stars), and MW (47). The period distributions of these stars are plotted in Figure 1.

The OGLE database does not distinguish between fundamental mode Cepheids and Cepheids pulsating in the first overtone

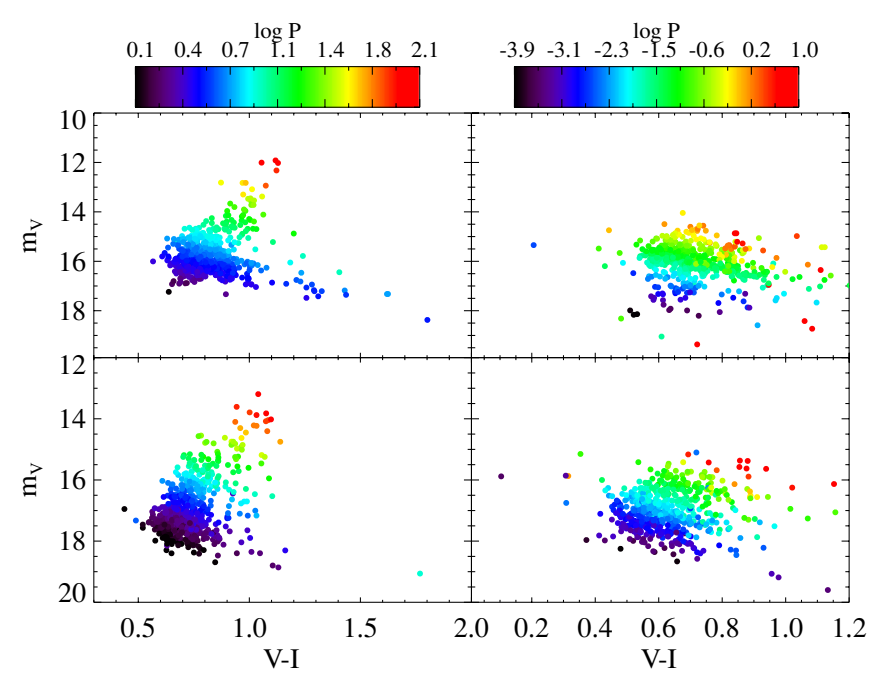

Figure 3. Color-magnitude plots for the OGLE stars, uncorrected for reddening. Points have been color-coded by pulsation period. The upper panels show LMC stars, while the lower panels show SMC stars. Fundamental mode pulsators are shown on the left and first overtones are on the right.

mode. Figure 2 shows our simple cuts in period-magnitude space used to provisionally classify OGLE stars as fundamental or overtone pulsators. For the rest of the stars, we use the source catalog designation of overtone or fundamental Cepheid. Color-magnitude diagrams of the OGLE are plotted in Figure 3.

We initially decompose each light curve in our sample into Fourier components by fitting the following equations to the $V$ and $I$ light curves simultaneously:

$$
\begin{aligned}
& m_{I}(t)=m_{I, 0}+\sum_{k=1}^{k=8} \alpha_{k} \sin (2 \pi k t / P)+\beta_{k} \cos (2 \pi k t / P), \\
& m_{V}(t)=m_{V, 0}+\sum_{j=1}^{j=8} \alpha_{j} \sin (2 \pi j t / P)+\beta_{j} \cos (2 \pi j t / P),
\end{aligned}
$$

where $P$ is the period, $t$ is the Julian day of the observation, $m_{I, 0}$ and $m_{V, 0}$ are the average magnitudes in each band, and the $\alpha$ and $\beta$ terms are the Fourier amplitudes. We constrain $\beta_{j=1}$ to zero to impose a common phase for all the fits. These
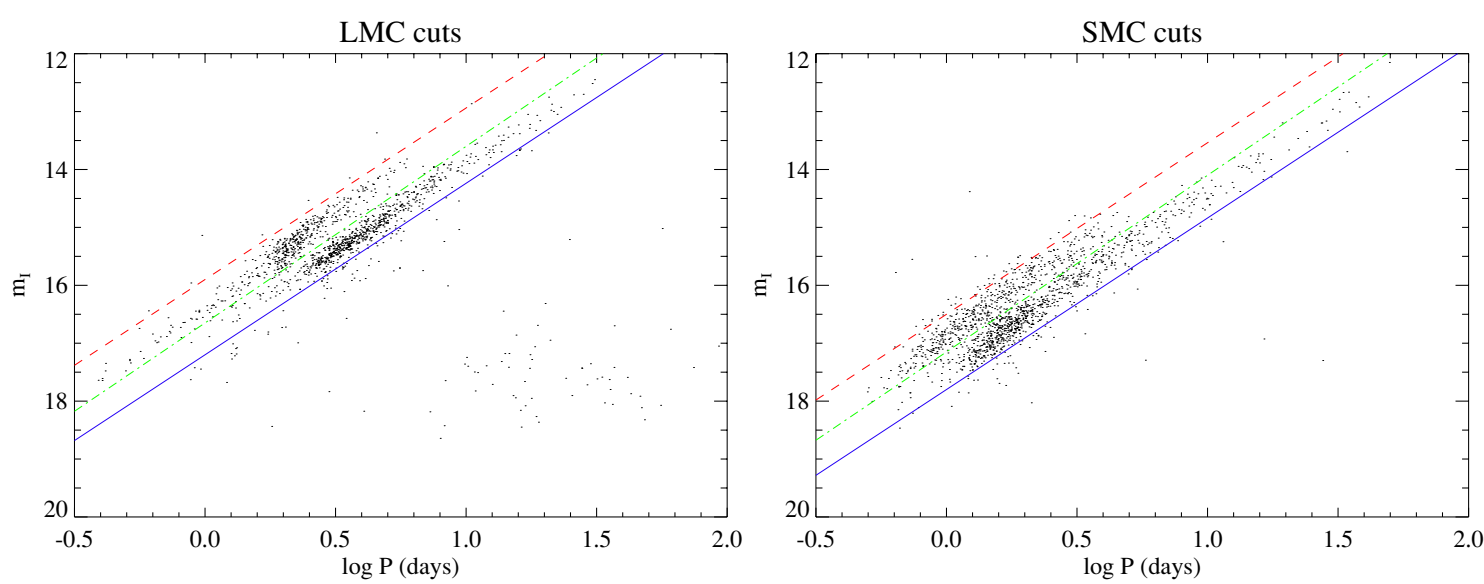

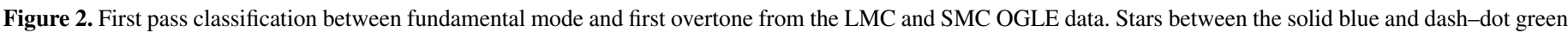

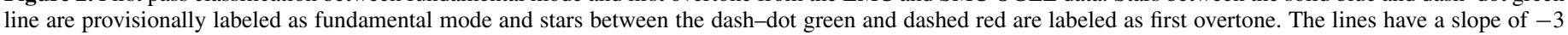
and $y$-intercepts of 17.2, 16.65, and 15.9 for the LMC and 17.8, 17.15, and 16.5 for the SMC. 


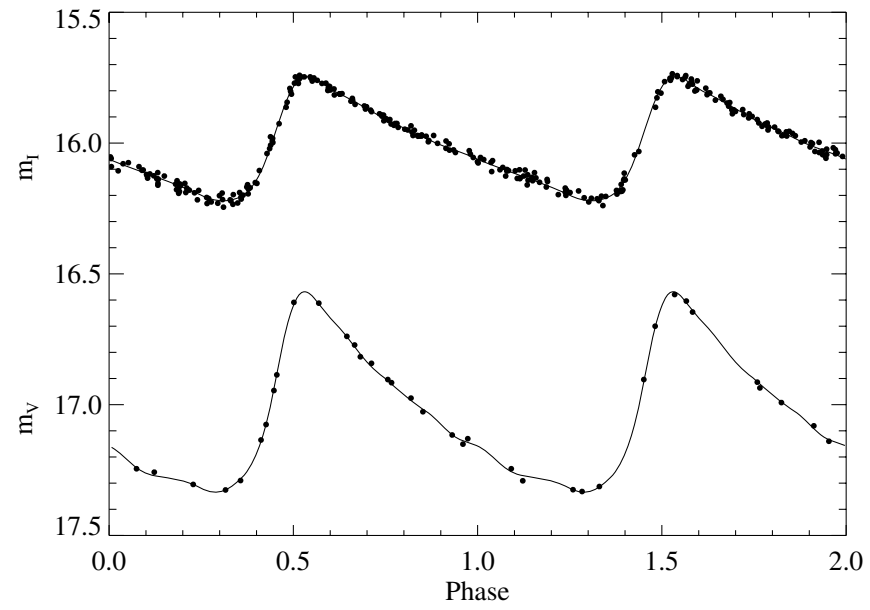

Figure 4. Example of an OGLE 2.8 day period Cepheid with the solid curves showing the best-fit Fourier decomposition. The upper curve shows the $I$ band, while the lower curve shows $V$. The sparse sampling in the $V$ band around a phase of 1.0 and 1.2 allows the fit slightly too much freedom (the curve rises slightly in those two regions). By performing the PCA analysis over many hundreds of light curves, such discrepancies should be averaged out.
Fourier fits generate the $32 \alpha$ and $\beta$ values for each star that are used in the PCA. While the shapes of the light curves are fitted simultaneously, the average magnitudes in each band are completely independent. This ensures that our light-curve templates are not dependent on the various dust corrections (or lack thereof) that have been applied in our different source catalogs.

Ngeow et al. (2003) discuss how fitting Fourier components to sparsely sampled data can result in poor fits (see their Figure 2(b) for an example of how Fourier decomposition can fail for sparsely sampled light curves). To keep our fits constrained to reasonable shapes, we create a smooth light curve by linearly interpolating each observed Cepheid to include 50 points evenly distributed across the full phase of the light curve. We then fit the Fourier components of this smoothed light curve, and use the results as the initial guess for the fitting of the observed data points. In the final fit, each Fourier component is allowed to change by a maximum of $20 \%$ from the smoothed fit parameters, thereby preventing divergences compared to the smooth fit. Figure 4 shows typical results for out fitted Fourier components. Figures 5 and 6 show the distribution of the 16 best fit $\alpha$ and $\beta$ values for all of the fundamental and overtone I-band light curves.
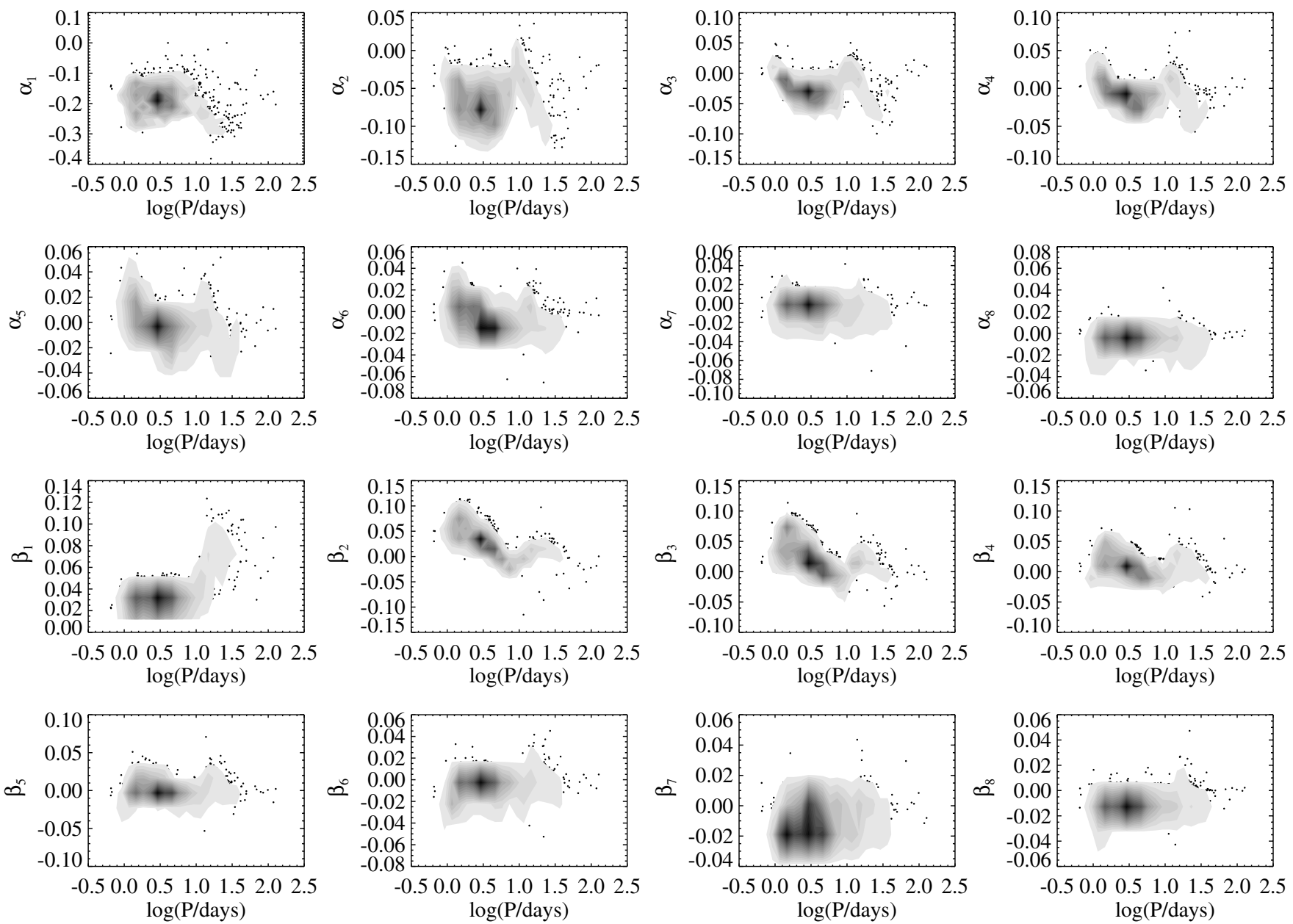

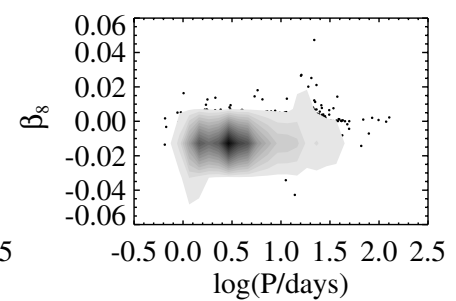

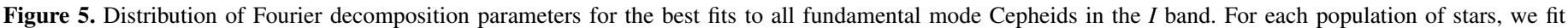

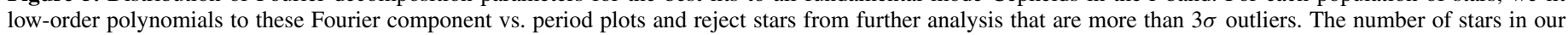
sample is large enough that we have plotted contours in the densely populated regions and individual points in the sparsely sampled regions. 

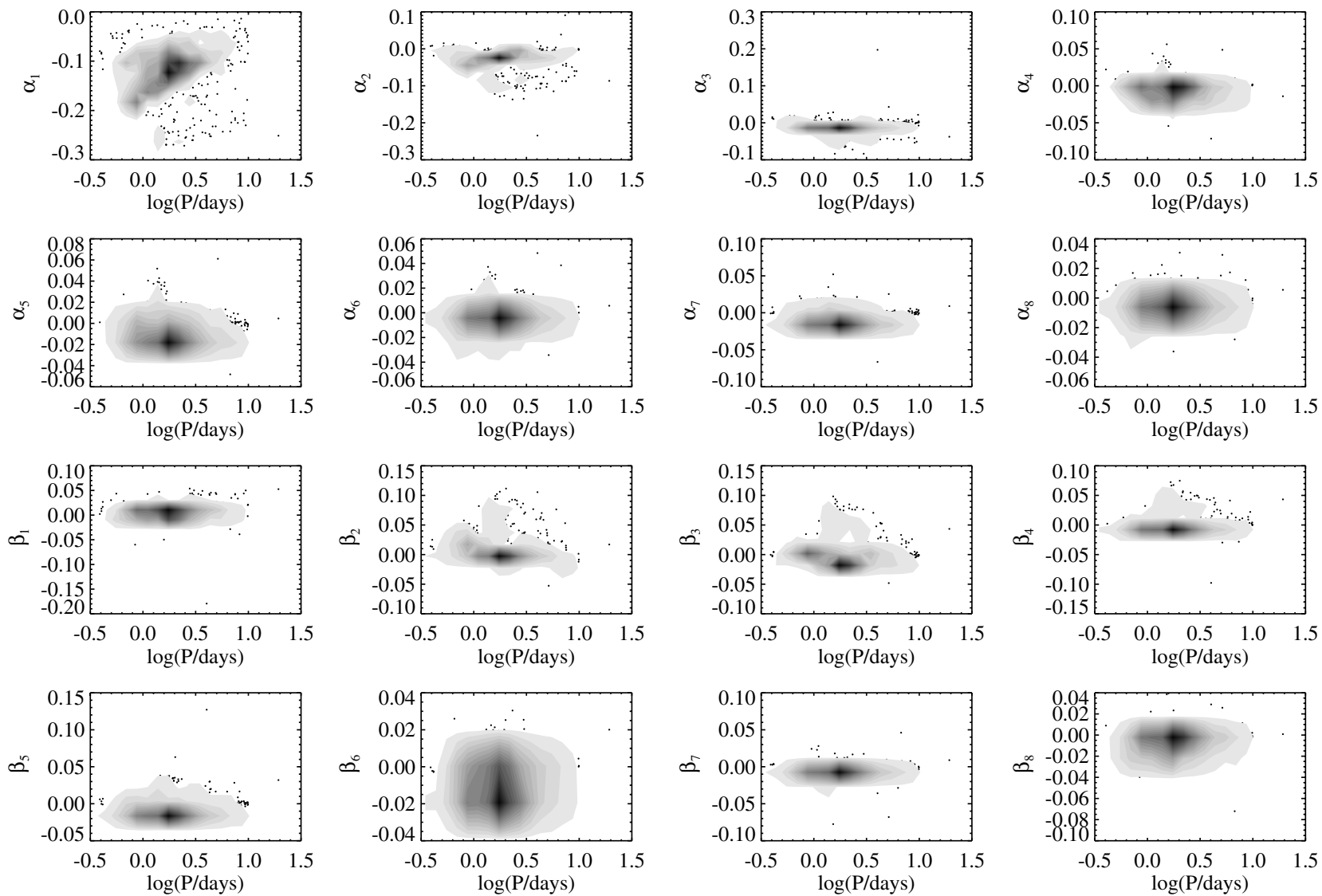

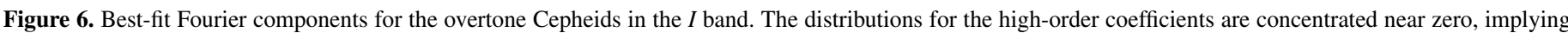
these light curves are simply sinusoidal, an unsurprising result for stars commonly referred to as "sinusoidal" or sCepheids.
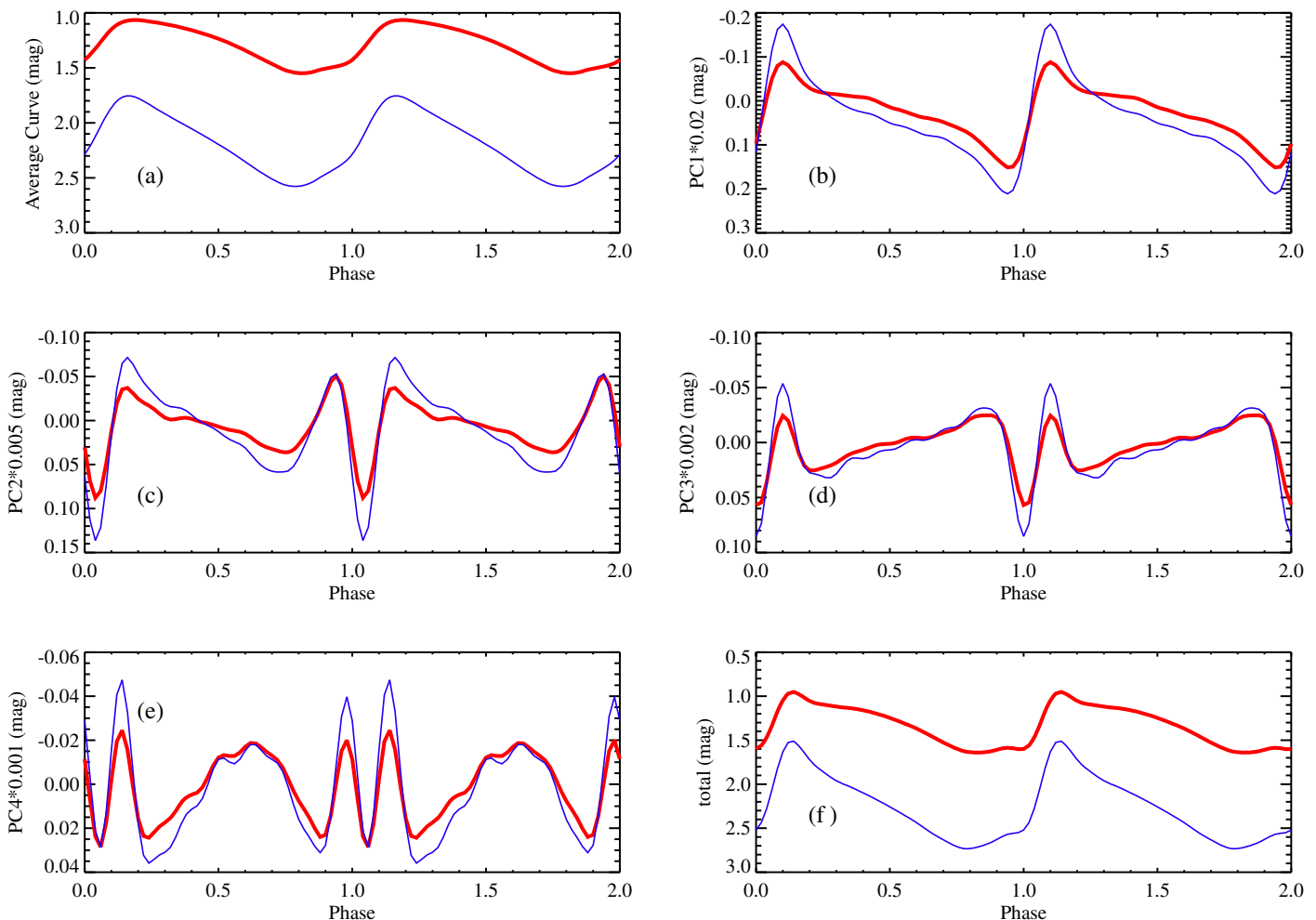

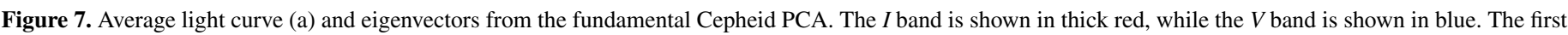

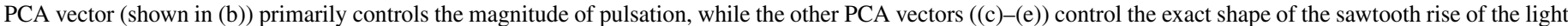

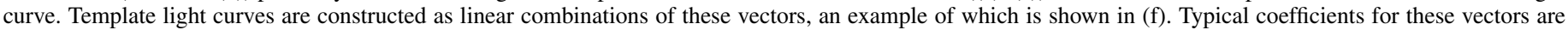
plotted in Figure 8. 

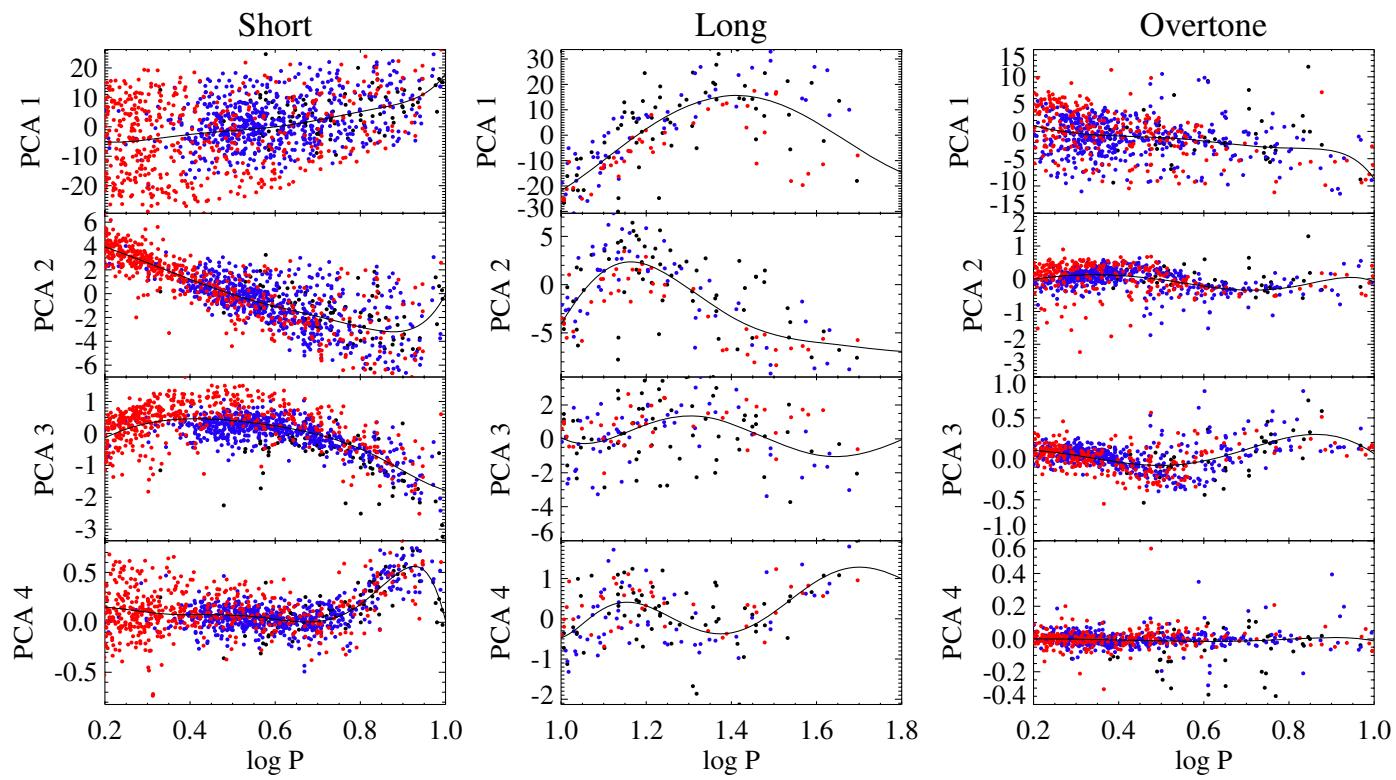

Figure 8. Template PCA coefficients (all multiplied by a factor of $10^{3}$ ) for all the fundamental mode Cepheids. The SMC, LMC, and MW stars are shown by red, blue, and black points, respectively. Our polynomial fits are plotted as solid curves. We have separated long-period, short-period, and overtone Cepheids into the left, middle, and right panels, respectively.

Table 1

Percentage of the Total Variance Contained in Each PCA Component for Each of our Constructed Templates

\begin{tabular}{lccccc}
\hline \hline Model & $\begin{array}{c}\text { PCA1 } \\
(\%)\end{array}$ & $\begin{array}{c}\text { PCA2 } \\
(\%)\end{array}$ & $\begin{array}{c}\text { PCA3 } \\
(\%)\end{array}$ & $\begin{array}{c}\text { PCA4 } \\
(\%)\end{array}$ & $\begin{array}{c}\text { Total } \\
(\%)\end{array}$ \\
\hline Short period & 70.1 & 18.7 & 4.8 & 1.4 & 95.1 \\
Long period & 64.1 & 16.6 & 6.8 & 2.9 & 90.4 \\
LMC & 66.7 & 15.6 & 9.8 & 1.8 & 94.0 \\
SMC & 70.1 & 17.2 & 6.0 & 1.5 & 94.8 \\
Overtones & 82.8 & 6.0 & 3.0 & 1.0 & 92.9 \\
\hline
\end{tabular}

\subsection{PCA Template Construction}

To test for differences in Cepheid populations, we constructed templates based on various subsets of the data. In particular, we made templates that include all our fundamental mode Cepheids and subsets that included only LMC stars, only SMC stars, only short-period stars, only long-period stars, and only overtones. For each subsample, we first subtracted an average light curve from each measured light curve. We then performed a PCA on the residuals to develop eigenvectors that can be used to correct for deviations from the average light curve. In all cases, over $90 \%$ of the variance could be described with only four principle component eigenvectors (Table 1). An example of an average light curve and PCA eigenvectors are plotted in Figure 7.

We plot the PCA vector strengths as a function of period in Figure 8. Several of the PCA vectors show strong trends with period. This is fortunate (and not too surprising), as it means while fitting for a star's period, we can simultaneously make an educated guess as to what the corresponding shape parameters should be. Figures 9 and 10 show how well our templates fit a variety of fundamental and first overtone stars.

Before performing PCA, we needed 32 Fourier components, as well as two magnitudes, a period, and a phase to accurately fit a Cepheid light curve. After performing PCA $>90 \%$ of the variation in the light-curve's shape can be described with just four eigenvector amplitudes. As a final step we note that the eigenvector amplitudes are strong functions of period, allowing us to make a quality template fit with only four free parameters

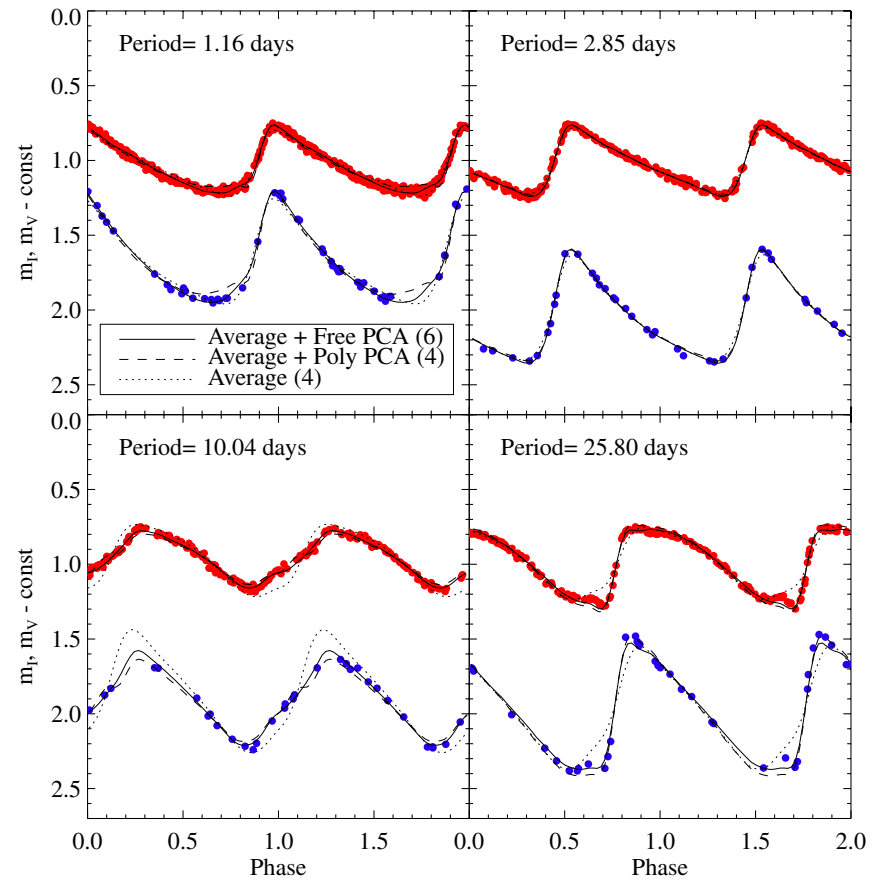

Figure 9. Examples of four OGLE fundamental mode Cepheids fit with our derived templates. Both the $V$ and $I$ light curves are fit simultaneously. The dotted lines show the best-fitting curve if we fit with just the average light curve (PCA eigenvectors fixed at zero), the dashed curves show the best fit if we set the PCA eigenvectors to the best polynomial fit values for the given period, the solid curves show the fit if we leave the first two PCA vectors as free parameters. The numbers in parentheses show the total number of free parameters in each fit.

(two average magnitudes, a phase, and a period). Figure 8 does show one possible pitfall as the first PCA vector for the shortperiod stars shows a great deal of scatter and little trend with period. We therefore caution that it is possible this eigenvector amplitude should be left as a free parameter if possible when fitting a light curve. 


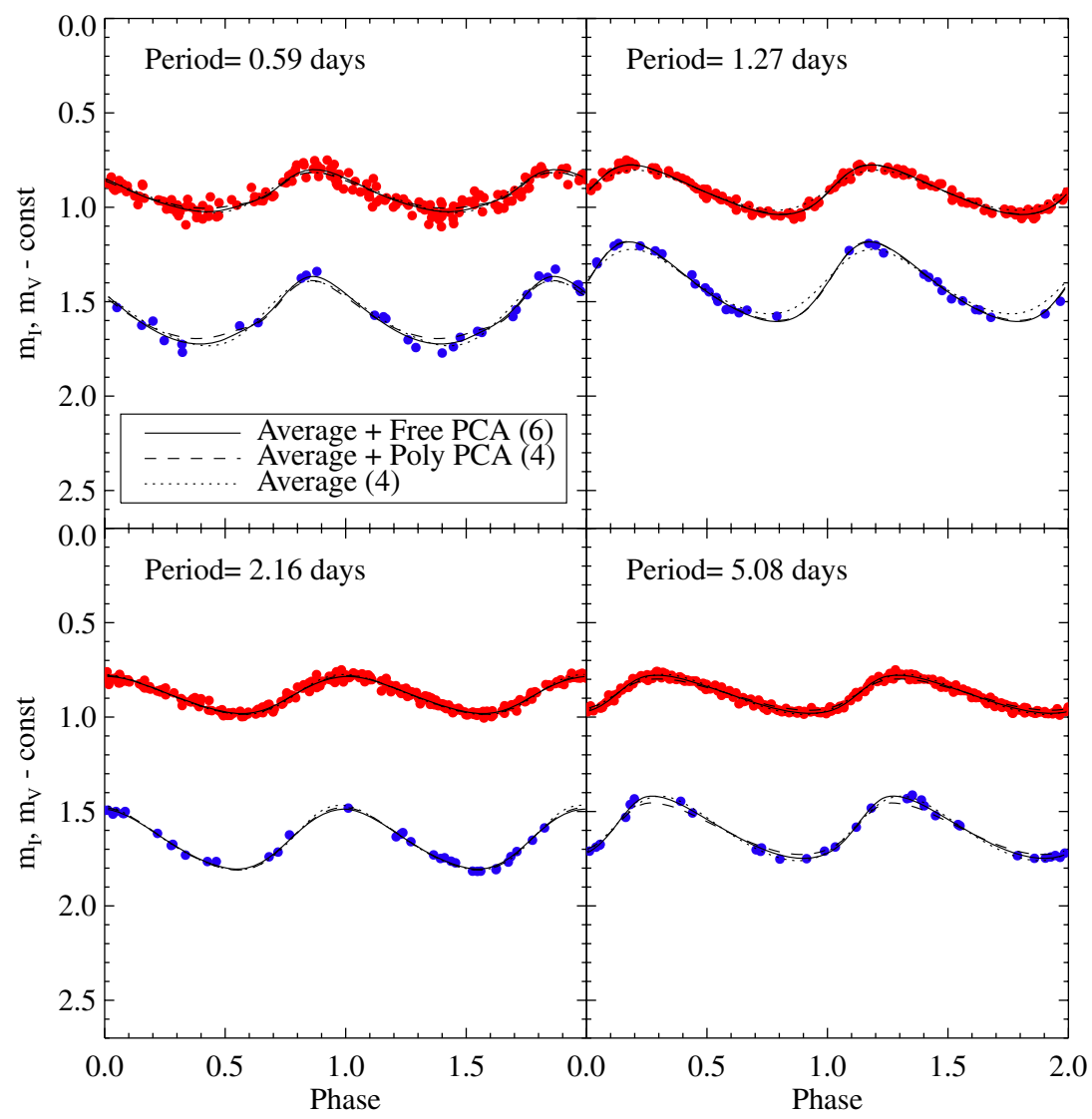

Figure 10. Same as Figure 9, only now for Cepheids identified as first overtones and fit with our overtone template. Again, it does not seem necessary to leave the PCA amplitudes as free parameters to converge on a quality fit.

Table 2

The Different Epoch Samplings Used in Our Monte Carlo Error Analysis

\begin{tabular}{lccc}
\hline \hline Model & $I$ Observations & $V$ Observations & Total \\
\hline 1 & 20 & 15 & 35 \\
2 & 10 & 5 & 15 \\
3 & 5 & 3 & 8 \\
4 & 4 & 2 & 6 \\
\hline
\end{tabular}

\section{TEMPLATE FITTING ACCURACY}

Tanvir et al. (2005) have already demonstrated that the template fitting technique is superior to other common period estimation techniques for cases where the photometry is noisy. They show magnitudes and periods determined through template fitting can reduce the scatter in distance estimates by $30 \%$ compared to simple string length methods.

We now endeavor to use Monte Carlo simulations to quantify how well our templates can recover magnitudes and periods from photometry of Cepheid stars. We have developed a fitting routine that uses Levenberg-Marquardt least-squares minimization to find the best-fitting Cepheid light curve, given a set of $V$ and $I$ photometry. The best-fitting template is found by varying period, phase, $\langle I\rangle$, and $\langle V\rangle$. We have included an option to vary the amplitude of the PCA eigenvectors. Fitting sparsely sampled light curves, there is a risk of aliasing or converging on local $\chi^{2}$ minima. To avoid such problems, we use a series of initial guess periods and phases to ensure we find the global $\chi^{2}$ minimum.

Our Monte Carlo varies four parameters to judge their impact on our fitting routine's robustness. First, we compare five well- sampled Cepheids from the OGLE database with different periods. Second, we vary the total number of observations in each band. Next, we look at possible effects of template mismatch (fitting LMC stars with a template derived from SMC stars, fitting overtone stars with fundamental mode templates and vice-versa). Finally, we vary the photometric precision of the light-curve points.

For each realization the star was randomly sampled in both bands. Therefore, there are $N_{I}+N_{V}$ unique epochs of observations and we do not explicitly model observing strategies that observe in multiple filters simultaneously.

We used four fundamental mode Cepheids (periods of 2.5, 4.2, 16.0, and 20.7 days), and one overtone (period 2.5 days) from the OGLE LMC data set for the Monte Carlo tests. All the photometry has initial errors of the order of $0.013 \mathrm{mag}$ in both bands. Although there could be some objection to using stars, which are included in the PCA analysis, our sample sizes are large enough that the addition or subtraction of a few stars should make little difference to our final templates.

For the error analysis, we fit for only period, $m_{v}, m_{I}$, and phase. We rely on the polynomial fits in Figure 8 to give reasonable amplitudes for the PCA eigenvectors. We also folded the original light curves so that the photometry covers at most three periods. This restriction is needed to keep the fitting procedure from falling into local minima caused by aliasing. When fitting sparsely sampled data over a long baseline a more brute-force exploration of period parameter space would be required than our current fitting procedure.

Figure 11 shows an example of one of our fitting simulations. As expected, the errors are largest for the overtone Cepheid when fit with the wrong template. We also show the calculated 


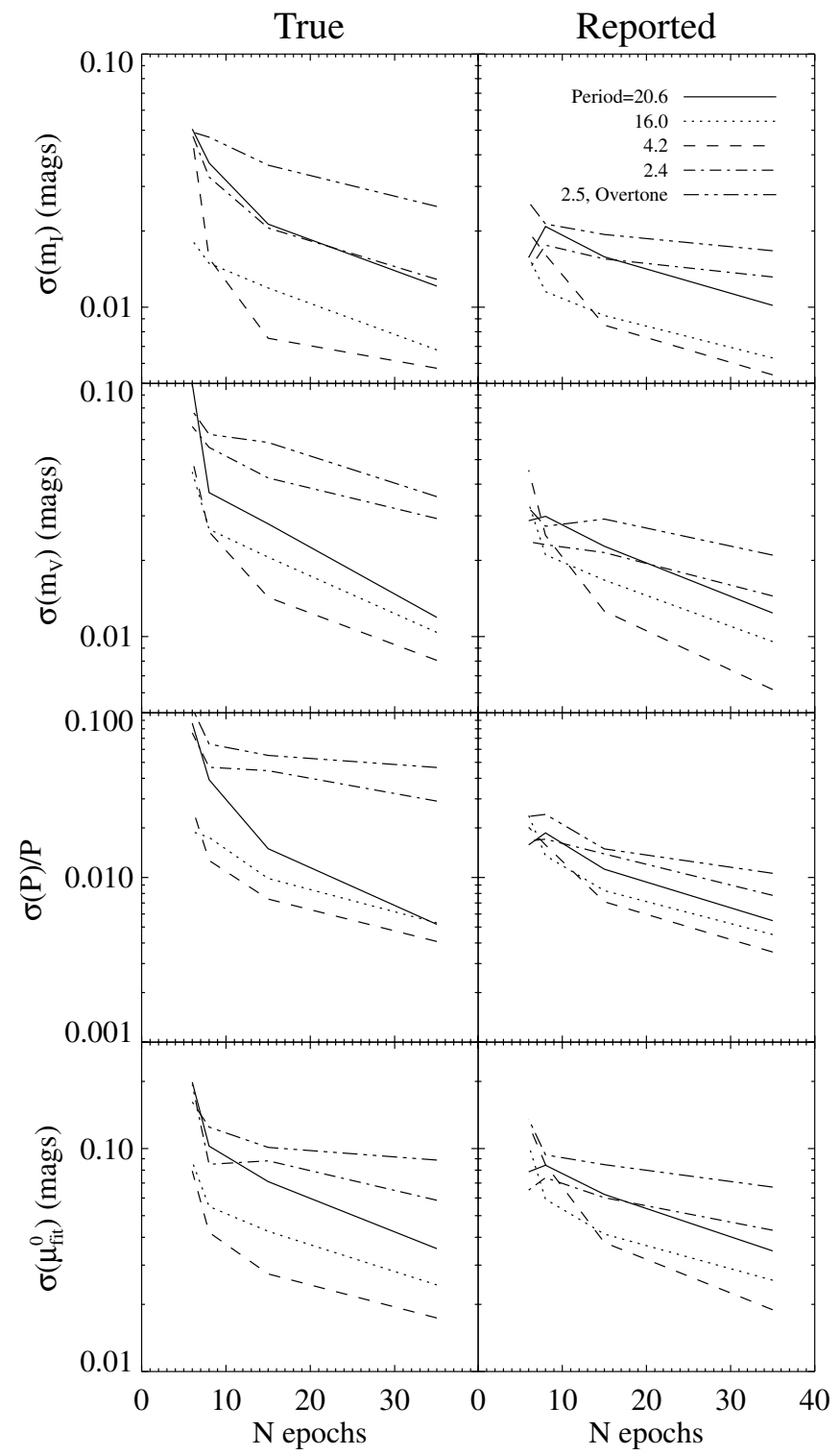

Figure 11. Results of our Monte Carlo simulation where we recover Cepheid properties using our templates and least-squares fitting procedure. We fit five different stars, along with the five different observing strategies listed in Table 2. The four panels on the left show the errors in fitting magnitudes and periods and the corresponding error in distance modulus. On the right, we show the uncertainties returned by our least-squares fitting routine. With photometric errors of $0.01 \mathrm{mag}$ and only six total epochs of observations, the uncertainties of the fitted parameters result in only a 0.1 mag uncertainty in $\Delta \mu_{\text {fit }}^{0}$.

uncertainties reported by our fitting routine in Figures 11 and 12. In general, the reported uncertainties are a good match to the actual errors resulting from the fits. The one exception is that the overtone uncertainties are underestimated as a result of making the assumption that the reduced $\chi^{2}$ should be unity, which is clearly incorrect in the case of fitting an overtone with a fundamental mode template. When we use an overtone template, there is a clear improvement in the $\chi^{2}$ values, indicating that it is a better fit.

We have also explored possible template mismatches due to metallicity by repeating the Monte Carlo experiment using an SMC template $([\mathrm{Fe} / \mathrm{H}] \sim-0.7)$ to fit LMC stars $([\mathrm{Fe} / \mathrm{H}] \sim$ $-0.3)$. The resulting periods and magnitudes are practically identical, suggesting that the templates can be used across different metallicity populations.

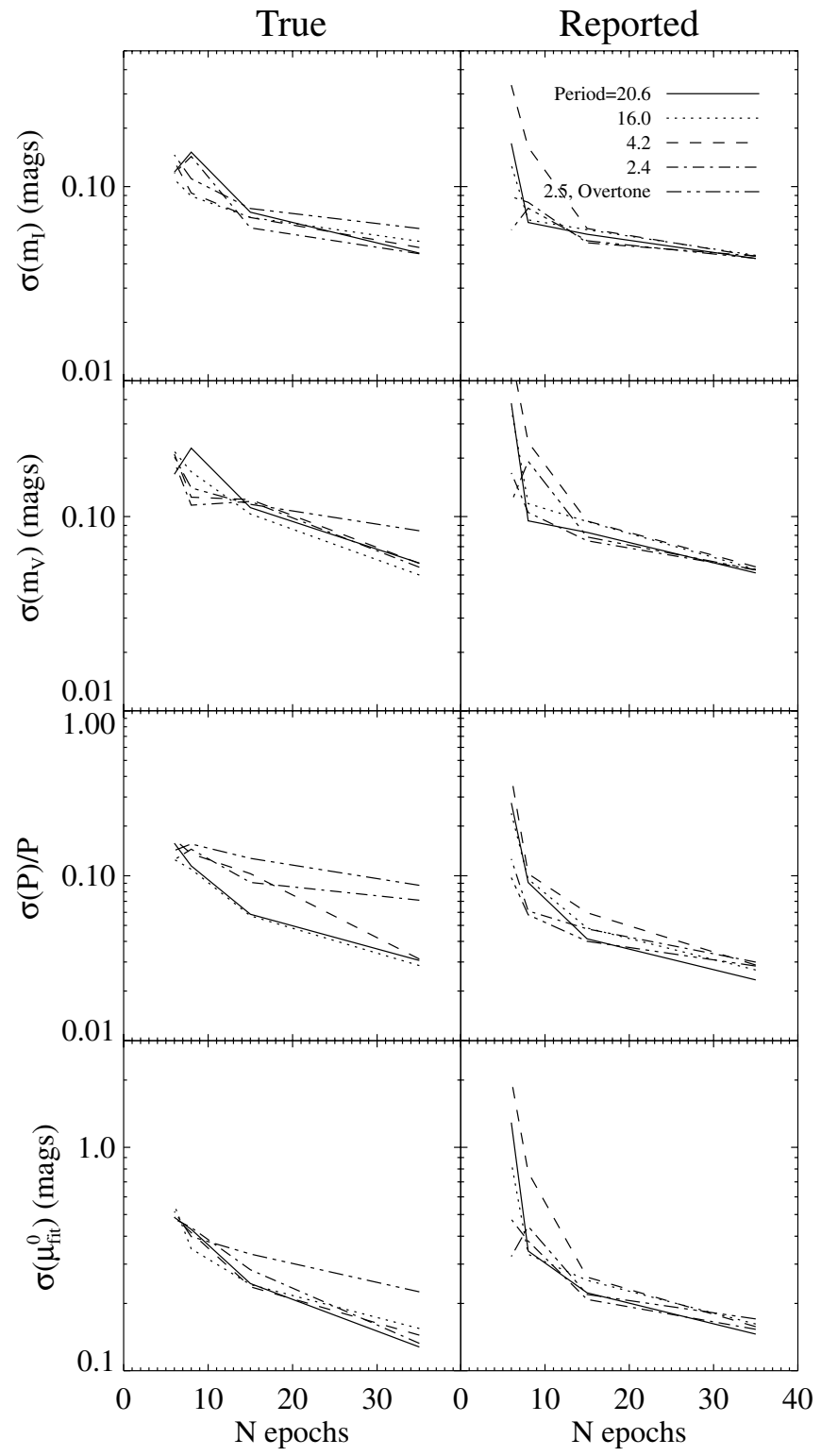

Figure 12. Same as Figure 11, only now the photometric errors have been increased to $0.2 \mathrm{mag}$. Again, the actual errors (left side) are very similar to the returned uncertainties (right side).

A proper assessment of the errors of individual measurements is key for determining a proper PL relation. We have therefore compared the uncertainties reported by our fitting procedure to the true offsets seen in our Monte Carlo resampling. Overall, the returned uncertainties are comparable to the actual errors derived from the Monte Carlo analysis. When the photometric errors were low $(0.01 \mathrm{mag})$, the returned uncertainties were slightly too small (by $\sim 15 \%$ ). On the other hand, when the errors were high and the sampling was sparse, the returned uncertainties were slightly larger than the true Monte Carlo calculated errors. Typically, around $10 \%$ of the fits would fail catastrophically, usually caused by aliasing or very sparse sampling. These failures could readily be seen as poor $\chi^{2}$ values or by visual inspection. This seems to imply that we can use the returned uncertainties, but as we will see later, observing strategy also affects the results and it is probably best to simply run a Monte Carlo for the photometric error and uncertainties for the specific sampling frequency used by a given observing program. 

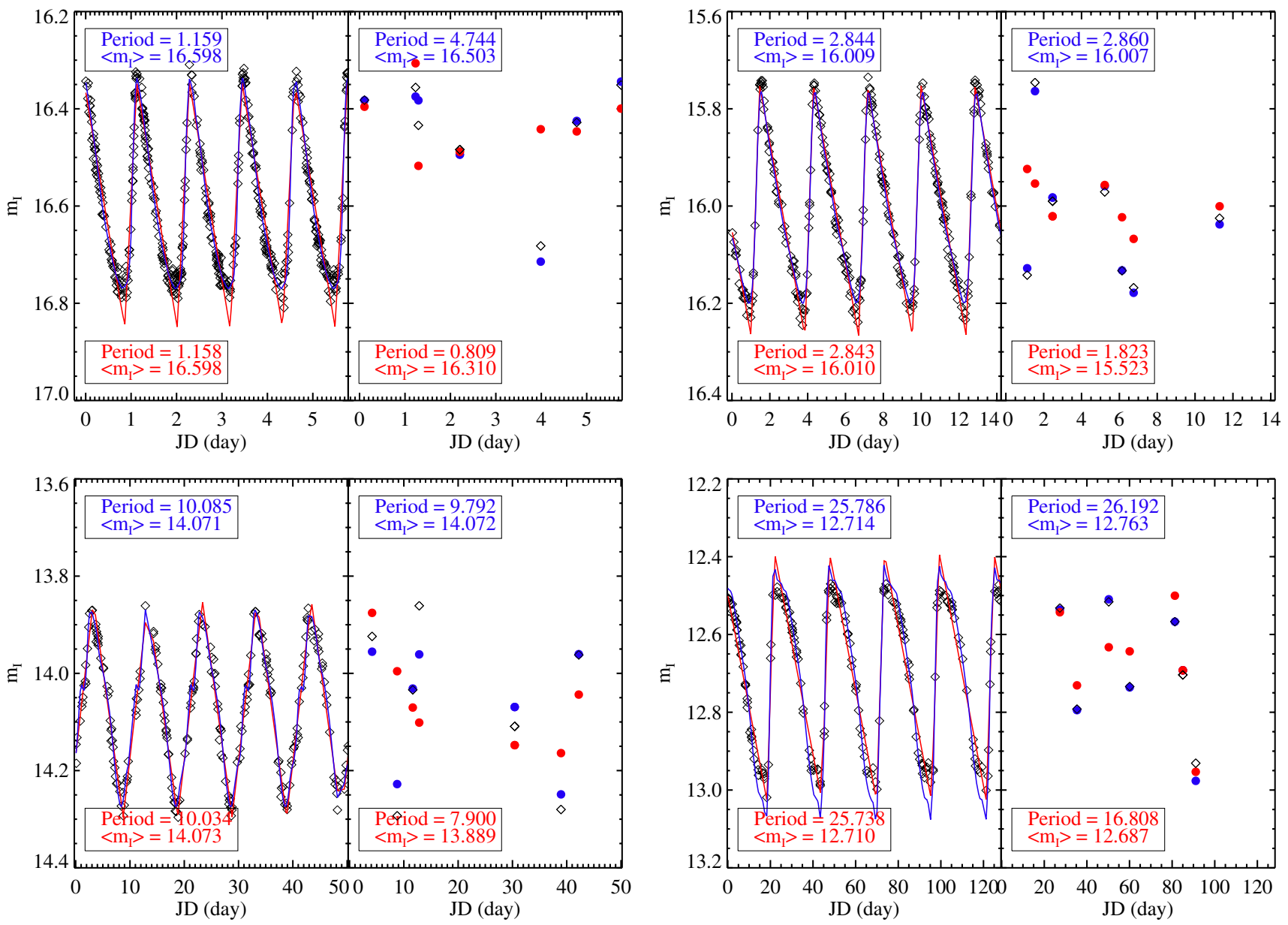

Figure 13. Example of how effective our templates can be at fitting sparsely sampled data. In the left-hand panels are the $I$-band light curves from Figure 9 fitted with our LMC templates (blue) and a simple asymmetric sawtooth function (red). The right-hand panel shows the results when seven random points from the light curve are sampled and the fitting is repeated. In four of the five cases, the templates are able to fit accurate average magnitudes and periods. The larger number of free parameters in the sawtooth function prevents it from properly converging in the sparsely sampled cases.

We ran several simulations to test how sensitive our fits are to the phase coverage of the observations. Generally, if the observations do not span more than half of the full phase, there is a large likelihood $(10 \%-40 \%)$ that the fitted period will catastrophically fail (defined as a final fitted distance error of greater than $10 \%$ ).

To illustrate how well our fitting procedure works, we compared our template fits to fits using a simple asymmetric sawtooth function. The results are plotted in Figure 13. When the light curves are well sampled, the templates and sawtooth converge to practically identical values. In the sparsely sampled case (only seven observational epochs), the templates return accurate fits in four out of five cases, while the sawtooth function fits fail in every case.

In summary, our tests indicate that our PCA template technique can fit periods with a precision of $\pm 0.1-0.3$ days with only six epochs and photometric precision of $0.01 \mathrm{mag}$. If the number of epochs increases to 15 days, our uncertainty drops to $\pm 0.03-0.2$ days.

\section{CONVERTING FITS TO DISTANCES}

Having established that our templates can accurately fit Cepheid periods and average magnitudes, we now point out some of the potential pitfalls in using fitted parameters to derive accurate distances. In theory, distances from single Cepheids can be averaged together to find a precise distance to a galaxy.
Besides the usual systematic errors associated with photometry, there are additional caveats that apply when observing a sample of Cepheids. (1) Cepheids used for a distance calculation must be above the completeness limit of the observations, otherwise, faint short-period stars will be undersampled and the final calculated distance will be biased (Sandage \& Carlson 1988; Freedman et al. 2001). (2) If the Cepheids do not sample the full range of the instability strip, they can be offset from a standard PL relation. Mager et al. (2008) discuss how the scatter in the PL relation in the outskirts of NGC 4258 (Macri et al. 2006) is greatly reduced because the Cepheids populate a limited region of the instability strip. (3) Finally, there is always the risk that a Cepheid may not be deblended from a nearby optical/physical companion. Blending is expected to bias Cepheid distance measurements to smaller values (Stanek \& Udalski 1999; Mochejska et al. 2000, 2004).

In a companion paper (McCommas et al. 2009), we have successfully applied our templates to HST data. Figure 14 shows some examples of how well the templates can fit noisy and suboptimally sampled data. The templates only start to fail for the star with the longest period, where only half of the full phase is observed.

\subsection{Metallicity Effects on Light-Curve Shape}

There is some question as to whether the shape of the light curve can be used to determine the metallicity of a Cepheid 

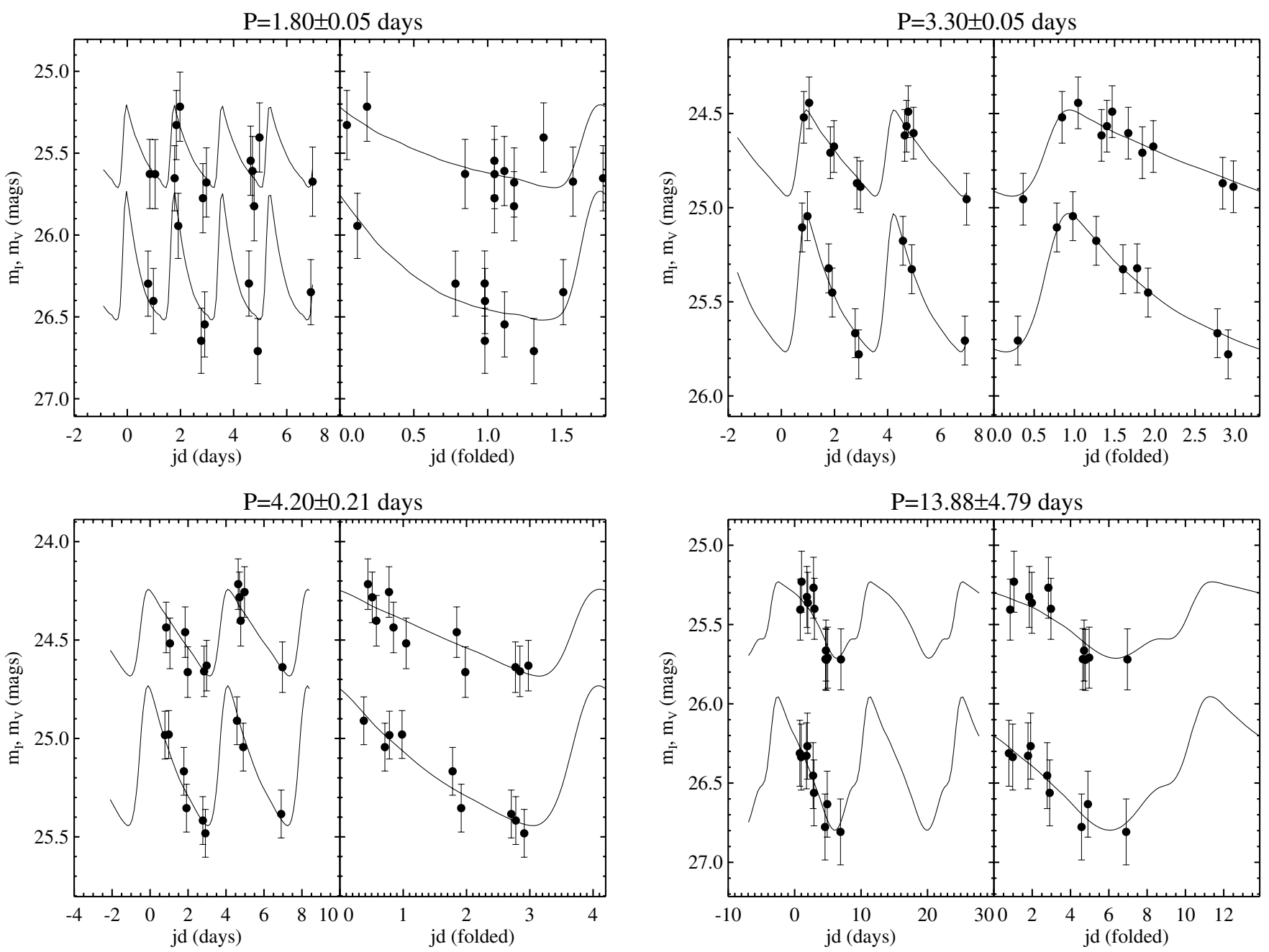

Figure 14. Example of $V$ and $I$ light curves of Cepheids in M81 observed with $H S T$ and fit with our templates. See McCommas et al. (2009) for a larger sample of Cepheids and a full distance calculation to M81. The fitted period in the lower right is poorly constrained due to the sparse phase coverage.

(Paczyński \& Pindor 2000; Kanbur et al. 2002). Looking at the long-period Cepheids (Figure 8), the first and second principle components have systematically smaller values for the SMC stars compared to the LMC and MW, suggesting that the shapes might be intrinsically different. We use a two-dimensional Kolmogorov-Smirnov test to compare the distributions in the PCA-period distributions (the MW, LMC, and SMC long-period variables all have very similar period distributions). The LMC and MW are consistent (probability $>0.12$ ) with being drawn from the same populations for all of the PCA vectors (i.e., the shape of the light curve at a given period does not show significant differences). The SMC shows a significant difference between the MW $(P=0.008)$ and $\operatorname{LMC}(P=0.04)$ in the distribution of the first PCA vector, but not the higher order PCA vectors. While the SMC light-curve shape is different on average, there is little hope of assigning a metallicity to an individual star based on its light-curve shape, since the intrinsic scatter within the SMC is larger than the differences from the LMC or MW. Even with the large number of stars in our sample, it is difficult to differentiate between the low-metallicity SMC Cepheids and the higher metallicity LMC and MW Cepheids. It is possible, in theory, to observe enough long-period stars that one could distinguish if a population was high or low metallicity based on the PCA distribution. However, it would require a prohibitively large sample size, as well as sufficient phase coverage that the shape parameters could be measured robustly.

Unlike the long-period variables, the short-period Cepheids in the three systems have very different period distributions. However, the PCA distributions overlap to a sufficiently large degree (Figure 8) that light-curve shape cannot readily be used to measure the metallicity of individual stars.

While we find metallicity does not alter light-curve shapes significantly, there is evidence that metallicity differences can alter the PL relation. Several studies claim to find a metallicity dependence (Kennicutt et al. 1998; Sakai et al. 2004; Macri et al. 2006; Saha et al. 2006; Romaniello et al. 2008; Sandage \& Tammann 2008), while others find that the PL relation is constant across galaxies (Udalski et al. 2001; Gieren et al. 2005).

\section{CONCLUSIONS}

We have extended the PCA techniques of Tanvir et al. (2005) to generate template light curves of Cepheid variables and first overtone variables. We have used a Monte Carlo simulation to demonstrate how robustly our templates can be used to fit Cepheid periods and magnitudes. Unlike previous studies, we do not limit ourselves to stars with periods longer than 10 days. Finally, we demonstrate the effectiveness of our templates on HST data and show that our techniques can be used to fit 
accurate periods and luminosities even when the observations have not been optimally spaced for observing variable stars. Our templates open up a new regime of distance measurement possibilities by enabling accurate fits for long-period, shortperiod, and overtone Cepheids from noisy and sparsely sampled observations.

We thank Andy Becker for helpful conversations. Thanks to Anil Seth and Greg Stinson for demanding more creative paper titles. P.Y. was supported by the Harlen J. Smith Postdoctoral Fellowship. J.J.D. and P.Y. were partially supported through NSF grant CAREER AST-0238683 and the Alfred P. Sloan Foundation. Support for this work was provided by NASA through grant G0-10915 and AR-10945 from the Space Telescope Institute, which is operated by the Association of Universities for Research in Astronomy, incorporated under NASA contract NAS5-26555. This work made use of the McMaster Cepheid database, maintained by Doug Welch. This work made use of Craig Markwardt's totally awesome IDL curve fitting code.

\section{REFERENCES}

Barnes, T. G., III, Fernley, J. A., Frueh, M. L., Navas, J. G., Moffett, T. J., \& Skillen, I. 1997, PASP, 109, 645

Berdnikov, L. N. 1997, VizieR Online Data Catalog, 2217, 0

Berdnikov, L. N., \& Turner, D. G. 1995, Pis ma Astronomicheskii Zhurnal, 21, 803

Berdnikov, L. N., \& Turner, D. G. 2001, ApJS, 137, 209

Burke, E. W., Jr., Rolland, W. W., \& Boy, W. R. 1970, J. R. Astron. Soc. Canada, 64, 353

Conselice, C. J. 2006, MNRAS, 373, 1389

Coulson, I. M., \& Caldwell, J. A. R. 1985, South African Astron. Obs. Circ., 9, 5

Deb, S., \& Singh, H. P. 2009, arXiv:0903.3500

Faber, S. M. 1973, ApJ, 179, 731

Freedman, W. L., et al. 2001, ApJ, 553, 47

Gieren, W. 1981, ApJS, 47, 315
Gieren, W., Storm, J., Barnes, T. G., III, Fouqué, P., Pietrzyński, G., \& Kienzle, F. 2005, ApJ, 627, 224

Henden, A. A. 1996, AJ, 111, 902

Kanbur, S. M., Iono, D., Tanvir, N. R., \& Hendry, M. A. 2002, MNRAS, 329, 126

Kennicutt, R. C., Jr., et al. 1998, ApJ, 498, 181

Lafler, J., \& Kinman, T. D. 1965, ApJS, 11, 216

Macri, L. M., Stanek, K. Z., Bersier, D., Greenhill, L. J., \& Reid, M. J. 2006, ApJ, 652,1133

Mager, V. A., Madore, B. F., \& Freedman, W. L. 2008, ApJ, 689, 721

McCommas, L. P., Yoachim, P., Williams, B. F., Dalcanton, J. J., Davis, M. R., \& Dolphin, A. E. 2009, AJ, 137, 4707

Mochejska, B. J., Macri, L. M., Sasselov, D. D., \& Stanek, K. Z. 2000, AJ, 120, 810

Mochejska, B. J., Macri, L. M., Sasselov, D. D., \& Stanek, K. Z. 2004, in ASP Conf. Proc. 310, IAU Colloq. 193, ed. D. W. Kurtz \& K. R. Pollard (San Francisco, CA: ASP), 41

Moffett, T. J., \& Barnes, T. G., III 1984, ApJS, 55, 389

Moffett, T. J., Gieren, W. P., Barnes, T. G., III, \& Gomez, M. 1998, ApJS, 117 135

Ngeow, C., \& Kanbur, S. 2008, arXiv:0805.4624

Ngeow, C.-C., Kanbur, S. M., Nikolaev, S., Tanvir, N. R., \& Hendry, M. A. 2003, ApJ, 586, 959

Ochsenbein, F., Bauer, P., \& Marcout, J. 2000, A\&AS, 143, 23

Paczyński, B., \& Pindor, B. 2000, ApJ, 533, L103

Romaniello, M., et al. 2008, A\&A, 488, 731

Saha, A., Thim, F., Tammann, G. A., Reindl, B., \& Sandage, A. 2006, ApJS, 165,108

Sakai, S., Ferrarese, L., Kennicutt, R. C., Jr., \& Saha, A. 2004, ApJ, 608, 42

Sandage, A., \& Carlson, G. 1988, AJ, 96, 1599

Sandage, A., \& Tammann, G. A. 2008, ApJ, 686, 779

Schaltenbrand, R., \& Tammann, G. A. 1971, A\&AS, 4, 265

Sebo, K. M., et al. 2002, VizieR Online Data Catalog, 214, 20071

Stanek, K. Z., \& Udalski, A. 1999, arXiv:astro-ph/9909346

Stetson, P. B. 1996, PASP, 108, 851

Tanvir, N. R., Hendry, M. A., Watkins, A., Kanbur, S. M., Berdnikov, L. N., \& Ngeow, C. C. 2005, MNRAS, 363, 749

Udalski, A., Soszynski, I., Szymanski, M., Kubiak, M., Pietrzynski, G., Wozniak, P., \& Zebrun, K. 1999a, Acta Astron., 49, 223

Udalski, A., Soszynski, I., Szymanski, M., Kubiak, M., Pietrzynski, G., Wozniak, P., \& Zebrun, K. 1999b, Acta Astron., 49, 437

Udalski, A., Wyrzykowski, L., Pietrzynski, G., Szewczyk, O., Szymanski, M., Kubiak, M., Soszynski, I., \& Zebrun, K. 2001, Acta Astron., 51, 221 